\title{
Economic Evaluation of Childhood 7-Valent Pneumococcal Conjugate Vaccination in Korea
}

\author{
Hyun Soon Sohn, PhD; Dong-Churl Suh, MBA, PhD; Eunjin Jang, PhD; and Jin-Won Kwon, PhD
}

\begin{abstract}
BACKGROUND: Streptococcus pneumoniae (sp) is a leading cause of invasive and noninvasive bacterial disease in children. 7-valent pneumococcal conjugate vaccine (PCV-7) has been shown to significantly reduce the incidence of pneumococcal diseases, such as meningitis, bacteremia, pneumonia, and otitis media. Although PCV-7 was introduced in Korea in 2003, it is not yet included in the universal immunization program.
\end{abstract}

OBJECTIVE: To evaluate the health outcomes, costs, and cost-effectiveness of universal vaccination with PCV-7 in Korean infants and to estimate the break-even price for PCV-7 from a societal perspective.

METHODS: A decision analytic model was used to evaluate the cost-effectiveness of immunization with PCV-7 in a birth cohort of Korean infants born in 2006. A universal vaccination strategy was compared with no vaccination in terms of costs and life years gained (LYG) over a 5-year time horizon. The birth cohort size, incidence of disease, resource utilization, and associated costs were obtained from the Korea National Statistical Office, the Korean Centers for Disease Control and Prevention, the Korean National Health and Nutrition Examination Survey, and the Korean Ministry of Health and Welfare. Inputs on the probabilities of clinical treatment pathways (e.g., tympanostomy) were derived from international literature if data specific to Korea did not exist. To estimate the benefits of universal immunization, the serotype-specific efficacy of PCV-7 was derived from studies conducted by Northern California Kaiser Permanente and by the Finnish Otitis Media Vaccine Study and applied to the serotypes isolated in Korean children with $\mathrm{sp}$ infections. The effects of vaccination on quality of life, herd immunity, benefits after the first 5 years of life, and patient copayments were not considered. A 4-dose schedule was used in the base-case analysis. A 3-dose schedule was also evaluated. The assumed price per dose was Korean won (KW) 70,000 (approximately US\$54; 2009 exchange rate US $\$ 1=K W 1,300$ ). Univariate and probabilistic sensitivity analyses were performed.

RESULTS: Implementing a 4-dose universal PCV-7 vaccination strategy in a birth cohort of 451,514 infants in Korea would prevent 96,728 cases of pneumococcal-related infections (591 meningitis, 1,379 bacteremia, 43,950 pneumonia, and 50,808 otitis media cases) and 218 deaths (199 discounted deaths averted, 575 discounted LYG over 5 years). The medical and nonmedical cost burden of pneumococcal diseases offset with vaccination was KW44,033 million (US\$33.87 million). The incremental discounted cost of universal vaccination was estimated to be KW86,384 million (US\$66.45 million). The incremental cost per LYG was KW150.2 million (US\$115,549) for the 4-dose schedule and KW103.91 million (US\$79,955) for the 3-dose schedule. The break-even costs were KW22,100 and KW28,100 per dose for the 4- and 3-dose schedules, respectively.

CONCLUSIONS: Universal PCV-7 vaccination of infants in Korea could substantially reduce pneumococcal disease morbidity, mortality, and related costs by preventing pneumococcal infections. However, at current market prices for the vaccine, a universal vaccination strategy is not cost-effective. The literature suggests that factors not considered in this analysis, including vaccine price reduction and indirect effects on public health (e.g., herd immunity), have the potential to make the public health impact and costeffectiveness of universal PCV-7 vaccination in Korea more favorable.

J Manag Care Pharm. 2010;16(1):32-45

Copyright $\odot$ 2010, Academy of Managed Care Pharmacy. All rights reserved.

\section{What is already known about this subject}

- The best way to prevent pneumococcal diseases is through vaccination. 7-valent pneumococcal conjugate vaccine (PCV-7) significantly reduced the incidence of pneumococcal diseases in large-scale studies conducted in the United States and Europe. PCV-7 is used in 74 countries worldwide and is incorporated into the national childhood immunization schedules in 16 countries. The World Health Organization recommends including PCV in infant vaccination programs.

- PCV-7 was introduced to Korea in 2003 but is not included in the universal immunization program mandated by the Korean Centers for Disease Control and Prevention. Only 15.3\% of infants were administered at least 1 dose of PCV-7 in 2006. Although cost and perceptions of cost-effectiveness may affect public health vaccine recommendations, to date no study has estimated the cost effectiveness of PCV-7 immunization in Korea.

- In many countries, vaccine cost was an important variable affecting the cost-effectiveness of including PCV-7 in nationwide immunization programs. Studies that evaluated benefits and costs of PCV-7 in the United States and Canada suggested that vaccine cost would need to be lower than the manufacturer's list price to achieve cost savings. More recent studies have also shown PCV-7 to be highly cost-effective when indirect effects on public health (e.g., herd immunity) are taken into account.

\section{What this study adds}

- This is the first health economic evaluation of universal PCV-7 immunization in Korean infants. A decision analytic model estimated that PCV-7 immunization with a 4-dose schedule in a 2006 birth cohort of 451,514 infants in Korea could prevent 96,728 cases of pneumococcal diseases and 218 deaths (199 discounted deaths averted; 995 discounted life years gained [LYG]) during the first 5 years of life.

- A universal PCV-7 vaccination program would initially cost Korean won (KW) 126,424 million (US\$97.3 million) to purchase the vaccine (KW70,000 [US\$54] per dose for 4 doses). Over a 5 -year time horizon, PCV-7 vaccination could reduce medical and nonmedical costs by KW44,033 million (US\$33.9 million), resulting in net program costs of KW82,391 million undiscounted (KW86,384 million discounted). The incremental costs per LYG were KW150.2 million (US\$115,549) and KW103.9 million (US\$79,955) for the 4- and 3-dose strategy, respectively.

- A PCV-7 vaccination program would result in cost savings if the vaccine price per dose was KW22,100 and KW28,100 or less for the 4- and 3-dose schedules, respectively. As in other countries, vaccine price is just 1 determinant of cost-effectiveness for a PCV-7 universal immunization strategy in Korea. The attractiveness of a PCV-7 program depends both on vaccine price and on the potential indirect effects that vaccination has on public health. 
S treptococcus pneumoniae (sp) is a leading cause of invasive and noninvasive bacterial disease in children throughout the world, including Korea. ${ }^{1,2}$ Pneumococcal diseases are treated primarily with penicillin, but penicillin resistance in nasopharyngeal $s p$ is high in healthy Korean children, with rates of $82 \%$ reported in recent years. ${ }^{3}$ The high rate of drug resistance makes treatment of pneumococcal infection difficult, possibly resulting in longer hospitalizations, use of more expensive alternative antibiotic therapy, higher mortality, and consequently increased medical costs. ${ }^{4}$ Because $s p$ is associated with bacterial meningitis, a disease with high mortality rates, careful selection of antibiotics and prevention strategies for these organisms is important. In Korea in 2006, the cost of pneumococcal diseases in children younger than 15 years of age was estimated to be Korean won (KW) 201 billion (US\$154.6 million; 2009 exchange rate US $\$ 1=\mathrm{KW1}, 300$ ). Across all age groups in Korea, the economic burden of pneumococcal diseases was estimated at KW790 billion (US\$607.7 million), including KW148 billion for otitis media, KW550 billion for pneumonia, and KW92 billion for both meningitis and bacteremia. ${ }^{1}$

The best way to prevent $s p$ diseases is through early vaccination. The 7-valent pneumococcal conjugate vaccine (PCV-7) has been available since 2000 to provide protection against pneumococcal diseases in children younger than 2 years of age. PCV-7 is considered clinically effective and safe and has shown significant reductions in the incidence rates of invasive pneumococcal disease, pneumonia, and otitis media in several large-scale studies conducted in the United States and Europe in infants and children. ${ }^{5-10}$ In the United States, where PCV-7 is used routinely to vaccinate children younger than 5 years of age, surveillance data from 2001 through 2003 documented substantial declines in invasive pneumococcal disease compared with pre-vaccine years. ${ }^{11} \mathrm{PCV}-7$ is currently available in 74 countries worldwide with 16 having incorporated PCV-7 into their national childhood immunization schedules. ${ }^{12}$ The World Health Organization (WHO) recently reported that pneumococcal disease is the foremost infectious disease among causes of death preventable by vaccination in children 5 years or younger worldwide and, as a result, has recommended including PCV in infant vaccination programs. $^{13}$

Pneumococcal nasal carriage was found in $9.3 \%$ of the healthy children aged 9 years or younger and in 30\%-34\% of outpatient and hospitalized children aged 5 years or younger in Korea. Among the isolated sp serotypes identified in these nasal carriages, $84 \%$ are covered by PCV-7. ${ }^{14-17}$ This finding suggests that PCV-7 vaccination may reduce nasal carriage of $s p$ and subsequent related pneumococcal infections. PCV-7 was introduced to Korea in late 2003. Because PCV-7 is an optional vaccination as recommended by the Korean Pediatric Society and is not part of the essential universal immunization program mandated by the Korean Centers for Disease Control and Prevention (KCDC), it is not covered by national health insurance. Lee et al. reported in 2006 that only 15.3\% of all Korean infants were administered at least 1 dose of PCV-7 within 7 years after birth. ${ }^{1}$

Many factors can affect public health vaccine recommendations and vaccination rates. One possible limiting factor may be cost and perceptions of cost-effectiveness. The price of PCV-7 per dose makes PCV-7 the most expensive component of the routine immunization schedule. ${ }^{18}$ In a decision analytic model of the cost-effectiveness of PCV-7, a universal vaccination program in the United States was estimated to save \$342 million in medical and $\$ 415$ million in nonmedical costs, such as lost work time, before accounting for the vaccine acquisition cost. ${ }^{19}$ At the manufacturer's current list price of $\$ 58$ per dose, infant vaccination would cost society $\$ 80,000$ per life-year saved. It was estimated that vaccination of healthy infants would result in net savings for society if the vaccine cost less than $\$ 46$ per dose and net savings for the payer if the vaccine cost less than $\$ 18$ per dose. ${ }^{19}$

The objectives of the present study were to estimate the projected health benefits, costs, and cost-effectiveness of universal routine vaccination in infants considered as the primary target group for PCV-7 in Korea. An additional aim was to determine the vaccine price at which the PCV-7 immunization program would break even.

\section{Methods}

\section{Decision Analytic Model Overview}

A decision analytic model was used to estimate the public health and economic impact of a universal PCV-7 strategy compared with no vaccination on a static birth cohort. This model assumed as a starting point that all Korean infants born in 2006 were vaccinated with PCV-7 following a 4-dose schedule. The cohort size (451,514 infants) was defined based on birth statistics (Korean Life Table) provided by the Korea National Statistical Office. ${ }^{20}$ The cohort was assumed to change annually on the basis of death rate at each age band reported on the Korean Life Table. A decision tree (Figure 1) was constructed to project the impact of infant vaccination on the incidence of invasive pneumococcal diseases including meningitis, bacteremia, pneumonia, and otitis media. The conceptual framework for this decision tree has been extensively reported in the literature..$^{19,21-23}$

The analyses were performed primarily from a societal perspective over a 5-year time horizon. Approximately $75 \%$ of invasive pneumococcal diseases in children occur before the age of 5 years. ${ }^{1}$ The efficacy of the vaccine for more than 5 years after immunization has not yet been determined. ${ }^{21}$ With uncertainty relating to the duration of vaccine protection, this model was designed to project the impact of PCV-7 on pneumococcal disease burden, associated costs, and life years gained (LYG) during the first 5 years of life. Costs and benefits were discounted at 5\%. Microsoft Excel 2000 (Microsoft Corp., Redmond, WA) was used to project health outcomes and costs, producing costeffectiveness analyses for 3- and 4-dose schedules, including univariate and probabilistic sensitivity analyses based on Monte Carlo simulation. In addition, break-even cost simulations were performed for 3- and 4-dose schedules. 


\section{FIGURE 1 Decision Analytic Model for PCV-7 Vaccination Program}

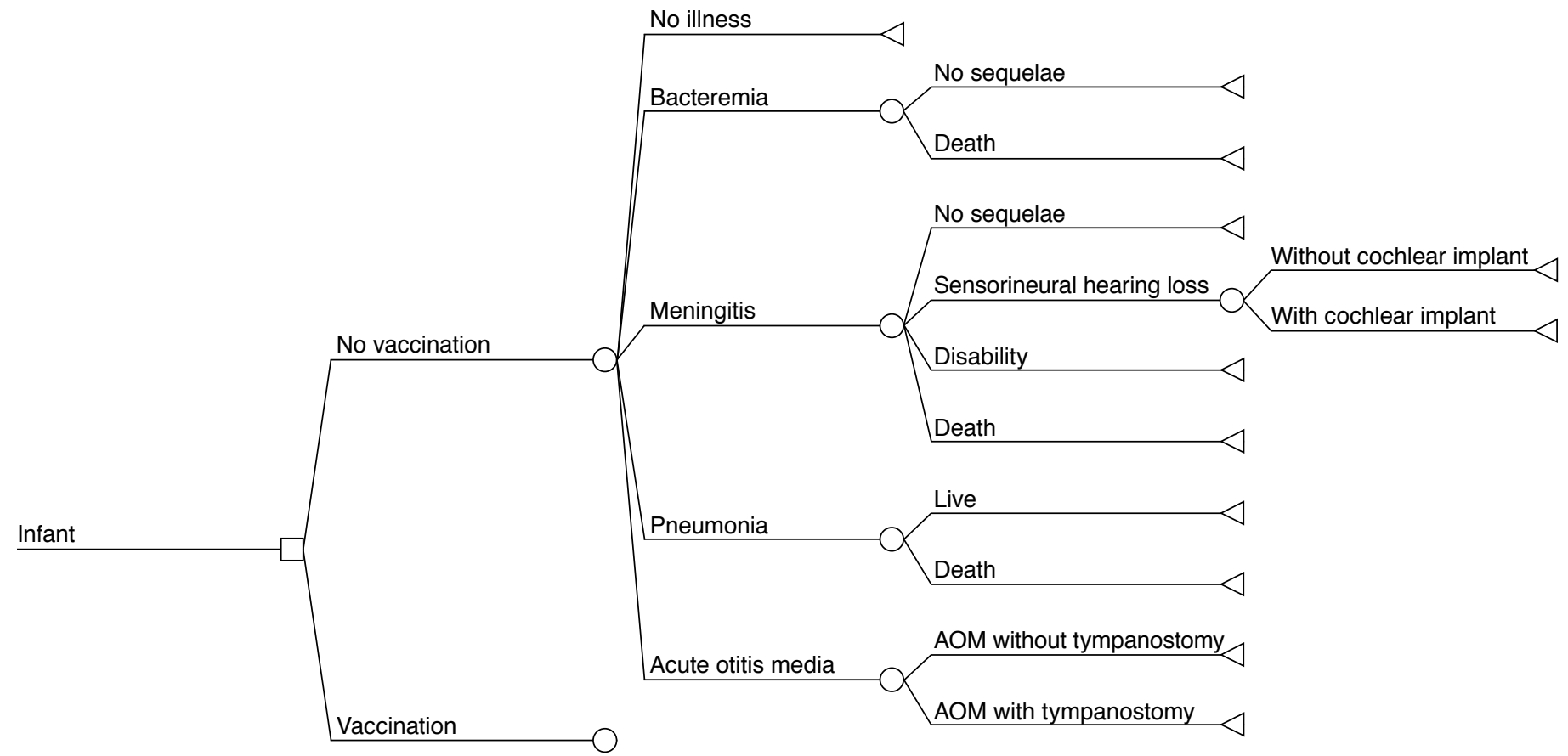

AOM = acute otitis media $P C V-7=7$-valent pneumococcal conjugate vaccine.

\section{Epidemiology of Pneumococcal Diseases}

The clinical and laboratory criteria used to establish estimates of pneumococcal disease incidence in this study varied according to the disease. Bacterial meningitis was based on WHO criteria, defined as having 1 or more clinical signs or symptoms of meningitis lacking an identifiable bacterial pathogen and 1 of the following: a cerebrospinal fluid (CSF) appearing turbid; reports of protein $>100$ milligrams per deciliter ( $\mathrm{mg}$ per $\mathrm{dL}$ ), glucose $<40 \mathrm{mg}$ per $\mathrm{dL}$; or white blood cell (WBC)/100 cells per cubic millimeter with $>80 \%$ neutrophils. ${ }^{24}$ For estimation of invasive pneumococcal disease incidence, we considered probable bacterial meningitis that was treated medically and resulted in medical costs based on a study conducted by Kim et al. (2004). ${ }^{24}$ Bacteremia was defined if the pathogen was isolated only in the blood without an identified focal lesion and with no symptoms other than those associated with bacteremia or sepsis. ${ }^{25}$

The long-term clinical sequelae associated with pneumococcal diseases were considered in this model. Meningitis can cause severe nervous system damage, such as hearing loss and paralysis. Neurosensory hearing loss is a common severe consequence of childhood bacterial meningitis. It can be a barrier to a child's normal speech, language, educational, and social development. ${ }^{26-27}$ Survival with deafness was assumed to require cochlear implants. ${ }^{28}$ Cochlear implantation has become the standard treatment for young children with severe to profound neurosensory hearing loss in Korea. ${ }^{29}$ We assumed tympanostomy tube insertion for $5.9 \%$ of patients with acute otitis media to allow drainage of the middle ear to improve hearing and reduce the pain caused by the pressure of the purulent effusion. ${ }^{8,30,31}$ Meningitis, bacteremia, and pneumonia could also result in death. However, we did not assume any mortality from otitis media.

Pneumococcal disease-related probabilities were derived from published data sources that included primarily Koreanspecific epidemiology data and clinical studies supplemented by national health statistic sources in Korea (Table 1). 1,8,20,24,31-39 We used pneumococcal disease incidence rates primarily from reliable Korean-specific reports and National Health Insurance (NHI) claims data reported by the Korean Health Insurance Review \& Assessment Service Agency (HIRA). Data from other countries were used when Korean data were not available. For example, the probability of a child receiving a tympanostomy as a consequence of otitis media came from non-Korean sources, such as the United States and Finland. ${ }^{8,31}$ The probabilities of the remaining clinical sequelae occurring from pneumococcal diseases and disease-related mortality were based on clinical studies of Korean children. ${ }^{1,24,32-39}$ 
TABLE 1 Model Inputs: Pneumococcal Disease-Associated and Vaccine EfficacyAssociated Variables in Korean Children Younger Than 5 Years of Age

\begin{tabular}{|c|c|c|c|}
\hline & Base-Case Value & $\begin{array}{c}\text { Ranges for } \\
\text { Sensitivity Analysis }\end{array}$ & Sources \\
\hline Birth cohort size & 451,514 & & Korea Statistical Information Service 20 \\
\hline \multicolumn{4}{|l|}{ Annual incidence (per 100,000) } \\
\hline Meningitis & 91.0 & $75.3-110.0$ & Kim et al. 200424 \\
\hline Bacteremia & $215.5^{\mathrm{a}}$ & $178.3-260.5$ & Kim et al. $2004,{ }^{24}$ Choi and Lee $1998^{36}$ \\
\hline Pneumonia & $11,988.2$ & Base $\pm 25 \%$ & HIRA $2006^{37}$ \\
\hline Otitis media & $20,951.8$ & Base $\pm 25 \%$ & HIRA $2006^{37}$ \\
\hline \multicolumn{4}{|l|}{ Rate of $s p$} \\
\hline Meningitis & 0.435 & & Lee et al. $2006^{1}$ \\
\hline Bacteremia & 0.429 & & Lee et al. $2006^{1}$ \\
\hline Pneumonia & 0.268 & & Lee and Woo $2007^{38}$ \\
\hline Otitis media & 0.292 & & Jung et al. $2000^{39}$ \\
\hline \multicolumn{4}{|l|}{ Annual probabilities of clinical sequelae } \\
\hline Disability from meningitis & 0.031 & & Kim et al. 200424 \\
\hline Severe hearing impairment from meningitis & 0.018 & & Lee et al. $2006,{ }^{1}$ Lee et al. $2007^{32}$ \\
\hline Cochlear implant among patients with severe hearing & 0.217 & & Ahn et al. $2007^{33}$ \\
\hline Tympanostomy from otitis media & 0.059 & & Fireman et al. $2003,{ }^{8}$ Palmu et al. 200431 \\
\hline \multicolumn{4}{|l|}{ Annual mortality } \\
\hline Meningitis & 0.046 & & $\begin{array}{l}\text { Lee et al. } 2006,{ }^{1} \text { Kim et al. } 2004,{ }^{24} \\
\text { Park et al. } 2000^{34}\end{array}$ \\
\hline Bacteremia & 0.043 & & Lee et al. 2006, ${ }^{1}$ Kim et al. $2005^{35}$ \\
\hline Pneumonia & 0.003 & & Lee et al. $2006^{1}$ \\
\hline \multicolumn{4}{|l|}{ Serotype coverage rates of PCV-7 } \\
\hline Meningitis and bacteremia & 0.682 & & $\begin{array}{l}\text { Lee et al. 2006, }{ }^{1} \text {, Kim et al. 2004, } \\
\text { Choi 2008, }{ }^{41} \text { Lee et al. 200342 }\end{array}$ \\
\hline Pneumonia & 0.676 & & Choi $2008^{41}$ \\
\hline Otitis media & 0.648 & & $\begin{array}{l}\text { Kim et al. 2004, }{ }^{3} \text { Kim et al. 2002,15 Choi 2008, } \\
\text { Lee et al. 2003,42 Choi et al. } 2006^{43}\end{array}$ \\
\hline \multicolumn{4}{|l|}{ Vaccine efficacy (reduction of diseases) in 4-dose scenario } \\
\hline VT meningitis and bacteremia & $97.4 \%$ & & Black et al. $2000^{5}$ \\
\hline VT pneumonia & $90.0 \%$ & & Black et al. $2000^{5}$ \\
\hline VT otitis media & $57.0 \%$ & & Black et al. $2000^{5}$ \\
\hline Vaccine efficacy in 3-dose scenario & $95 \%$ of 4 doses & & Whitney et al. $2006^{49}$ \\
\hline Discount rate for outcomes (\%) & 5 & 3,7 & HIRA recommendation \\
\hline
\end{tabular}

Disease Incidence Rates. The incidence of meningitis was based on a large prospective, population-based surveillance study of invasive bacterial diseases in Korean children aged less than 5 years. ${ }^{24}$ Many of the infectious disease cases in Korea have negative culture results because antibiotics are often started empirically prior to obtaining cultures. In a 1998 Korean study of hospitalized children diagnosed with meningitis, only $18.0 \%$ of the cases were confirmed by both clinical symptoms and CSF tests. ${ }^{40}$ This problem has implications for our study because the true incidence of meningitis could be greater than the 91.0 per 100,000 we used in our calculations to estimate disease burden; sensitivity analyses were performed to address this issue. We assumed that the bacterial meningitis cases that met the WHO definition would receive clinical treatment. The incidence of bacteremia was calculated based on the proportions of bacteremia and meningitis cases among invasive pneumococcal disease cases reported in a recent large observational study ${ }^{36}$ The incidence rates of pneumonia and otitis media were estimated from NHI claims data. ${ }^{37}$ We estimated otitis media incidence on a per patient basis rather than a per episode basis. ${ }^{37}$

Causative $s p$ Rates. Determination of $s p$ as the underlying cause of pneumococcal disease was based on 3 Korean studies., ${ }^{1,38-39}$ In these studies, $s p$ was the underlying cause of $43.5 \%$ of meningitis, $42.9 \%$ of bacteremia, $26.8 \%$ of pneumonia, and $29.2 \%$ of otitis media cases.

Case Estimates of Morbidity and Mortality in an Unvaccinated Population. The numbers of cases of specific pneumococcal diseases were calculated by multiplying the specific disease incidence by the cohort size at each age-band and the rate of causative 
sp (Table 1). For example, the annual number of all-cause cases of meningitis without vaccination was estimated by multiplying the annual rate of cases (91.0 cases per 100,000 children) by the birth cohort size $(91.0 \div 100,000 \times 451,514=411)$ for the first year. The annual number of meningitis cases due to $s p$ was estimated by multiplying the annual number of all-cause cases by the rate of disease attributable to $s p(0.435 \times 411=179)$. The annual number of meningitis cases due to $s p$ calculated per each age was then summed over 5 years to reflect the total number of cases occurring during the first 5 years of life for the birth cohort.

To estimate the consequences of pneumococcal disease due to $s p$, the number of $s p$-related cases of disease was multiplied by the annual probability of each clinical sequela to generate an estimate of the number of infants with (a) disability or severe hearing loss from meningitis; (b) tympanostomy in patients with otitis media; and (c) mortality from meningitis, bacteremia, or pneumonia (Table 1). For example, the total number of deaths due to meningitis over the 5-year time horizon was 41 (the accumulated number of meningitis cases due to $s p$ [890] $\times$ the annual meningitis mortality rate [0.046]). To estimate the number of children receiving cochlear implants, the number of patients with severe hearing impairment due to meningitis was multiplied by the probability of receiving an implant.

\section{Vaccine Efficacy}

Multivalent conjugate vaccine has been proven to be immunogenic in infants, inducing immunologic memory and the formation of antibodies detectable in mucosal secretions and reducing nasopharyngeal carriage of pneumococci. ${ }^{6}$ The reported efficacy of vaccines can vary depending on which serotypes are responsible for the disease, vaccination schedules, epidemiology in a specific country, and ethnic differences in immunogenicity. The serotypes commonly found in Korea differ from those in the United States and Western Europe, where PCV-7 was developed to cover the pneumococcal serotypes frequently encountered there. Vaccine efficacy is dependent on the overlap of serotypes included in vaccines and serotypes of disease-causative sp. The efficacy of vaccines reported in other countries was applied in this model with adjustments for the different pneumococcal serotype distributions in Korea. Serotypes isolated in Korean children with pneumococcal infections were mainly 4,6A, 6B, 9V, 14, 19A, 19F, 23A, and 23F. ${ }^{17}$ The serotypes covered in PCV-7 include 4, 6B, 9V, 14, 18C, 19F, and 23F. Thus approximately $65 \%-68 \%$ of all serotypes isolated from cases of pneumococcal diseases in Korea are covered by PCV-7.1-3,15,41-43

This model assessed both a 4- and 3-dose vaccination schedule. The 4-dose schedule of the vaccine (at 2, 4, 6, and 12 months) is more closely aligned with the current immunization schedule guidelines recommended by the KCDC. ${ }^{44}$ There have been some reports of efficacy in partially vaccinated groups; for example, Kayhty et al. (2005) found that 3 doses of PCV-7 evoked strong antibody response for all serotypes. ${ }^{45}$ Pneumococcal serum antibody concentrations in patients treated with a 3-dose schedule were comparable to those with a 4-dose schedule. ${ }^{45-46}$ Vaccinations are scheduled as 4 doses in the United States, Canada, and Netherlands, whereas many European countries, such as Sweden, Denmark, Norway, Italy, and Finland, and countries in other regions, such as Australia, primarily have a 3-dose vaccination scheduled at 3, 5, and 12 months on the basis of immunogenicity studies. ${ }^{2,47}$ An immunogenicity study for PCV-7 in Korean infants reported that $97 \%-100 \%$ with a 3-dose vaccination showed a serum antibody concentration level greater than 0.35 micrograms per $\mathrm{mL}$ (the WHO-recommended minimum level to prevent invasive pneumococcal infections). Because post-vaccination mean serum antibody concentration levels were found to be higher in Korean infants than in the United States and European countries, ${ }^{48}$ we assumed that a 3 -dose vaccination schedule would provide preventive effects comparable to those of the 4-dose strategy. Thus, the immunogenicity studies and regulatory agencies appear to support a 3-dose vaccination schedule as an alternative to a 4-dose schedule.

The incidence of pneumococcal infections in vaccinated infants was assumed to be reduced in proportion to the demonstrated vaccine efficacy against infections. Serotype-specific efficacy rates were applied because vaccine efficacy against pneumococcal diseases is specific to 7 serotypes. No studies to our knowledge have evaluated PCV-7 vaccine efficacy in Korean children. There is, however, no evidence of ethnic variation in PCV-7 effectiveness. ${ }^{7}$ Therefore, assumptions about vaccine efficacy were derived from the largest studies currently available, including the U.S. Northern California Kaiser Permanente (NCKP) ${ }^{5}$ and the Finnish Otitis Media Vaccine (Finnish OM) ${ }^{6}$ studies. The NCKP study evaluated the efficacy, safety, and immunogenicity of PCV-7 vaccination at 2, 4, 6, and 12-15 months in a prospective doubleblind study among 37,830 children in a health maintenance organization. ${ }^{5}$ The Finnish study was a prospective, randomized, double-blind cohort design to evaluate PCV for the prevention of pneumococcal acute otitis media. ${ }^{6}$

We estimated pneumococcal disease cases that were averted by PCV-7 during the study period (Table 1). ${ }^{13,5,15,41-43,49}$ First, we estimated the number of $s p$-pneumococcal disease cases in Korea with serotypes covered by PCV-7. The serotype coverage rates ranged from $64.8 \%$ of otitis media to $68.2 \%$ of meningitis/bacteremia cases. Next, we multiplied the cases with PCV-7 serotype coverage by the vaccine's efficacy for pneumococcal diseases, assumed to be $97.4 \%$ for meningitis and bacteremia, $90.0 \%$ for pneumonia, and $57.0 \%$ for otitis media, using the 4-dose schedule. The estimated number of cases prevented by vaccination was equal to the serotype coverage rate times the efficacy rate times the estimated number of cases attributable to $s p$. For example, the estimated number of meningitis cases prevented by vaccination was $0.682 \times 0.974 \times 890=591$. The 3 -dose schedule was assumed to have $95 \%$ of the efficacy of the 4 -dose schedule. Mean values across studies were used for the probability of tympanostomy, 
Economic Evaluation of Childhood 7-Valent Pneumococcal Conjugate Vaccination in Korea

TABLE 2 Medical and Nonmedical Cost-Associated Variables in Korean Children Younger Than 5 Years of Age

\begin{tabular}{|c|c|c|c|}
\hline & Base Value & Ranges for Sensitivity Analysis & Sources \\
\hline Vaccine cost per dose $(\mathrm{KW}+000)$ & 70 & $10-70$ & Market price 52 \\
\hline \multicolumn{4}{|l|}{ Direct medical cost (annual average per patient) $(\mathrm{KW}+000)$} \\
\hline Meningitis & 357 & Base $\pm 25 \%$ & HIRA $2006^{37}$ \\
\hline Bacteremia & 337 & Base $\pm 25 \%$ & HIRA $2006^{37}$ \\
\hline Pneumonia & 190 & Base $\pm 25 \%$ & HIRA $2006^{37}$ \\
\hline Otitis media & 74 & Base $\pm 25 \%$ & HIRA $2006^{37}$ \\
\hline Hospitalized meningitis & 861 & & HIRA $2006^{37}$ \\
\hline Hospitalized bacteremia & 746 & & HIRA $2006^{37}$ \\
\hline Hospitalized pneumonia & 690 & & HIRA $2006^{37}$ \\
\hline Hearing loss & 320 & & HIRA $2006^{37}$ \\
\hline Disability & 1,698 & & HIRA $2006^{37}$ \\
\hline Cochlear implant & 705 & & Korea NHI reimbursement price list ${ }^{53}$ \\
\hline Tympanostomy & 149 & & Korea NHI reimbursement price list ${ }^{53}$ \\
\hline \multicolumn{4}{|l|}{ Hospitalization rate } \\
\hline Meningitis & 0.391 & & HIRA $2006^{37}$ \\
\hline Bacteremia & 0.460 & & HIRA $2006^{37}$ \\
\hline Pneumonia & 0.191 & & HIRA $2006^{37}$ \\
\hline Otitis media & 0.090 & & HIRA $2006^{37}$ \\
\hline \multicolumn{4}{|l|}{ Average annual physician visit frequency per outpatient } \\
\hline Meningitis & 2.0 & & HIRA $2006^{37}$ \\
\hline Bacteremia & 2.5 & & HIRA $2006^{37}$ \\
\hline Pneumonia & 4.0 & & HIRA $2006^{37}$ \\
\hline Otitis media & 6.5 & & HIRA $2006^{37}$ \\
\hline \multicolumn{4}{|l|}{ Average length of stay per hospitalized patient (days) } \\
\hline Meningitis & 6.7 & & HIRA $2006^{37}$ \\
\hline Bacteremia & 6.7 & & HIRA $2006^{37}$ \\
\hline Pneumonia & 7.7 & & HIRA $2006^{37}$ \\
\hline Otitis media & 4.5 & & HIRA $2006^{37}$ \\
\hline Transportation cost for hospitalized patient (1-way) (KW) & 11,019 & & Korea NHANES 54 \\
\hline Transportation cost for outpatient (1-way) (KW) & 8,891 & & Korea NHANES54 \\
\hline Hourly average wage for women aged 30-39 years (KW) & 8,946 & & Korea Statistical Information Service 20 \\
\hline Discount rate for cost (\%) & 5 & 3,7 & HIRA recommendation \\
\hline
\end{tabular}

HIRA = Health Insurance Review \& Assessment Service Agency; KW= Korean won; NHANES = National Health and Nutrition Examination Survey; NHI=national health insurance.

annual mortality for meningitis and bacteremia, and serotype coverage rates because these parameters were based on 2 or more studies.

Vaccine efficacy was assumed to be the same throughout 5 years and applied during the analysis period. ${ }^{49}$ In a recent economic study, PCV-7 was assumed to elicit a long-lasting immune response in young children, based on immunologic studies indicating that PCV-7 would stimulate a long-lasting antibody response and provide an extended duration of protection. ${ }^{50-51}$ However, vaccine effectiveness was not reported for each age group, and duration of protection afforded by the vaccine still remains uncertain. Thus, we assumed that protection efficacy would last at the same level during the 5 years.

\section{Costs}

The medical and nonmedical cost parameters used in this analysis are reported in Table 2. The average unit costs reported for medical and nonmedical cost items were based on Korean-specific data sources. ${ }^{20,37,52-54}$ Direct medical costs included the cost of vaccine and treatment for pneumococcal diseases. Direct nonmedical costs included transportation costs for medical care due to pneumococcal diseases. Indirect medical costs (not shown) included the costs associated with economic productivity loss (i.e., time spent caring for infected children by caregivers) owing to pneumococcal disease morbidity. Productivity loss due to premature death in nonvaccinated infants and productivity gains owing to prevention of premature death in vaccinated infants were not considered. These factors were not included to avoid double-counting the vaccination benefit and because children younger than 5 years of age are not old enough to participate in the labor force. In the vaccinated group, it was assumed that direct medical and nonmedical costs would be reduced to the same degree as vaccine-attributable prevention of diseases. All costs are reported 
in $2006 \mathrm{KW}$ currency. Prices were adjusted using the Korean Consumer Price Index. ${ }^{20}$

Vaccination Program Costs. The cost of PCV-7 is the only input cost for a vaccination program included in this model. We assumed that PCV-7 uptake was 100\% for all vaccination schedules in the 2006 birth cohort during the first year. The vaccine was assumed to be administered concurrently with other vaccines on the same day based on convenience and because there is no evidence of interactions between PCV-7 and other vaccines. ${ }^{12,55}$ The market price of PCV-7 vaccine per dose in Korea is KW100,000.52 Based on the Yearbook of Imported Pharmaceutical Goods ${ }^{56}$ and the importer's profit margin, we assumed a wholesale price of KW70,000 (approximately \$US54) per dose. We used the wholesale price in our base-case analysis because it better reflects actual cost in Korea than does the market price. Vaccinationrelated costs, such as administration, supplies, and medical staff time, were not considered because we assumed that PCV-7 was injected at the same office visit with other vaccines. Because vaccination safety data indicate that no local infusion-related reaction or side-effect was severe enough to require significant medical attention or treatment, ${ }^{48}$ these costs were not included in the model.

Medical Costs for Pneumococcal-Associated Diseases. Direct medical costs for individual pneumococcal diseases and clinical sequelae, such as hearing loss and disability, were derived from the 2006 NHI Yearbook. ${ }^{37}$ Average annual costs per patient were calculated as total NHI costs, excluding payments made by patients, divided by the total number of patients. Direct medical costs included medical resources, such as prescribed medications, medical procedures, and diagnostic tests, used by patients diagnosed and treated for pneumococcal diseases in inpatient, ambulatory, or emergency room settings. The costs for cochlear implants and tympanostomy tube insertions were based on the NHI Reimbursement Price List ${ }^{53}$ and multiplied by a defined weighted price factor of 1.25 because these procedures are typically administered in the hospital. We assumed that the cost for neurological disability in survivors of meningitis was similar to that of paralysis. The costs of disability and severe hearing loss from meningitis and cochlear implants and tympanostomy tube insertions were added to average annual disease cost.

Nonmedical Costs Including Transportation and Work Loss. Transportation costs for a medical institution visit were based on 2005 Korean National Health and Nutrition Examination Survey (NHANES III) data. ${ }^{54}$ The one-way costs for transportation for an outpatient and a hospitalized patient were reported to be KW8,891 and KW11,019, respectively. Time lost at work by the parent of a child with pneumococcal disease was valued using the human capital method. The average gross hourly wage for Korean women laborers aged 30-39 years (assumed to be the age group with children younger than 5 years of age) was reported to be KW8,946 per hour based on national data provided by the Korea National Statistical Office. ${ }^{20}$ We assumed 4 hours of work loss for an outpatient visit and 8 hours of work loss for an inpatient admission. The rates of hospitalization, frequency of outpatient visits, and length of stay for hospitalized patients were based on the NHI Yearbook. ${ }^{37}$ Transportation and time costs related to physician visits for vaccine administration were not included because we assumed that this vaccine was administered concurrently with other vaccines in the same visit.

\section{Analyses}

Pneumococcal disease cases and deaths for vaccination and no-vaccination strategies were compared to determine cases of pneumococcal disease and deaths prevented over a 5-year period from vaccination. The incremental cost and benefits of a direct vaccine-induced protection effect compared with no vaccination were calculated by adding costs and benefits for each of the 2 strategies. Incremental cost-effectiveness ratios (ICERs), expressed as cost per death averted and cost per LYG, were calculated for vaccination versus no vaccination. Differences in overall cost and outcomes between the vaccination and no vaccination scenarios, respectively, were calculated for the denominator and numerator of the ICER. A 5\% discount rate was applied to cost and benefits in the cost-effectiveness analyses.

Sensitivity Analyses. We evaluated how the model's results changed when key assumptions were varied over plausible ranges using univariate and probabilistic sensitivity analysis primarily around the cost per discounted death estimates. We performed sensitivity analyses for the following parameters: pneumococcal disease incidence rates, disease-associated medical costs, discount rate, and vaccine price per dose. The ranges of variables used in sensitivity analyses for the annual incidence of meningitis and bacteremia were based on the 95\% confidence intervals reported in published sources. A plus/minus $( \pm$ ) $25 \%$ range around the base-case value was applied for all other variables considered in the sensitivity analyses. We varied vaccine costs from KW10,000 up to KW70,000 (the base-case price) per dose. Additionally, a break-even cost, defined as the per dose vaccine price that results in a total cost of vaccination equivalent to the total resulting discounted cost savings for avoided pneumococcal disease, was calculated.

A probabilistic sensitivity analysis was performed using a 10,000-replication Monte Carlo simulation. We assumed that (a) incidence rates of pneumococcal diseases, such as bacteremia, meningitis, pneumonia, and otitis media, had beta-distributions; and (b) medical costs for bacteremia, meningitis, pneumonia, and otitis media and clinical sequelae, such as hearing loss and disability, had gamma-distributions, ${ }^{57}$ with standard errors that were the same as the mean values derived from the NHI Yearbook. We calculated 95\% confidence intervals (CI) for ICERs using the percentile method. Cost-effectiveness acceptability curves were calculated to identify the probability of vaccination 
Economic Evaluation of Childhood 7-Valent Pneumococcal Conjugate Vaccination in Korea

\section{TABLE 3 Estimated Pneumococcal Disease Outcomes and Costs in a Cohort of Korean Infants During the} First 5 Years of Life with No Vaccination and Vaccination, and ICER in a Base-Case Analysis

\begin{tabular}{|c|c|c|c|c|}
\hline \multicolumn{2}{|c|}{ Pneumococcal Disease Outcomes and Costs } & \multirow{2}{*}{$\begin{array}{c}\text { No Vaccination (NV) } \\
890\end{array}$} & \multirow{2}{*}{$\frac{\text { Vaccination }(\mathrm{V})}{299}$} & \multirow{2}{*}{$\frac{\text { Difference (NV-V) }}{-591}$} \\
\hline \multirow[t]{14}{*}{ Cases $^{\mathrm{a}}$} & Pneumococcal meningitis & & & \\
\hline & Pneumococcal bacteremia & 2,076 & 697 & $-1,379$ \\
\hline & Pneumococcal pneumonia & 72,238 & 28,289 & $-43,950$ \\
\hline & Pneumococcal otitis media & 137,558 & 86,749 & $-50,808$ \\
\hline & Subtotal & 212,762 & 116,034 & $-96,728$ \\
\hline & Disability from pneumococcal meningitis & 28 & 9 & -19 \\
\hline & Hearing loss from pneumococcal meningitis & 16 & 5 & -11 \\
\hline & Cochlear implant from pneumococcal meningitis & 3 & 1 & -2 \\
\hline & Tympanostomy from pneumococcal otitis media & 8,116 & 5,118 & $-2,998$ \\
\hline & Death from pneumococcal meningitis & 41 & 14 & -27 \\
\hline & Death from pneumococcal bacteremia & 89 & 30 & -59 \\
\hline & Death from pneumococcal pneumonia & 217 & 85 & -132 \\
\hline & Subtotal & 347 & 129 & -218 \\
\hline & Discounted total life years in birth cohort & $2,044,180$ & $2,044,378$ & $199^{b}$ \\
\hline \multirow[t]{5}{*}{ Costs (KW million)a } & Vaccine cost & 0 & 126,424 & 126,424 \\
\hline & Medical cost & 26,350 & 12,978 & $-13,372$ \\
\hline & Nonmedical cost & 69,134 & 38,473 & $-30,661$ \\
\hline & Subtotal & 95,484 & 177,875 & 82,391 \\
\hline & Discounted total net cost & 86,823 & 173,207 & 86,384 \\
\hline \multirow[t]{3}{*}{ Base-case analysis } & (at a vaccine price KW70,000 per dose) & \multicolumn{3}{|c|}{ ICER (KW million per LYG) } \\
\hline & 4-dose schedule & \multicolumn{3}{|c|}{435 per death averted; 150.2 per LYG } \\
\hline & 3-dose schedule & \multicolumn{3}{|c|}{301 per death averted; 103.9 per LYG } \\
\hline
\end{tabular}

being cost-effective at various levels of willingness to pay (WTP) for an additional discounted death averted.

\section{Results}

\section{Base Case Analysis}

The health outcomes and costs for the no-vaccination and universal vaccination strategies are presented in Table 3. Over a 5-year time horizon, the estimated numbers of cases of pneumococcal disease attributed to $s p$ in the 2006 birth cohort were 212,762 in the no-vaccination strategy and 116,034 under a universal 4-dose vaccination program. Permanent disability from meningitis was estimated to occur in 28 children without vaccination and 9 children with vaccination. Without vaccination, hearing loss from meningitis was estimated to occur in 16 children, with 3 of these receiving a cochlear implant. With vaccination, hearing loss and cochlear implant cases would be reduced to 5 and 1 , respectively. Tympanostomy was estimated to be performed in 8,116 children without vaccination and 5,118 with vaccination. The estimated numbers of deaths due to sp-related meningitis, bacteremia, and pneumonia were 347 in the no-vaccination strategy and 129 in a universal vaccination program. Thus, a 4-dose PCV-7 immunization program could prevent 96,728 cases of pneumococcal diseases (591 meningitis, 1,379 bacteremia, 43,950 pneumonia, and
50,808 otitis media) and 218 deaths, resulting in 575 discounted LYGs for the first 5 years from birth.

The medical and nonmedical cost burden of $s p$ pneumococcal disease in an unvaccinated birth cohort aged 5 years or younger was KW95,484 million. An estimated total of KW44,033 million in medical $(\mathrm{KW} 13,372)$ and nonmedical $(\mathrm{KW} 30,661)$ cost reductions would occur because of the 4-dose vaccine program. The 3-dose vaccine regimen would be expected to provide 95\% of the health and economic benefit of the 4-dose regimen based on our assumption of vaccine efficacy. The costs of acquiring vaccine for the 451,514 infants in the 2006 birth cohort were estimated to be KW126,424 million for the 4-dose regimen and KW94,818 million for the 3-dose regimen. The undiscounted incremental program costs were estimated to be KW82,391 million (discounted KW86,385 million) for the 4-dose regimen and KW52,986 million (discounted KW56,781 million) for the 3-dose regimen. For the 4-dose schedule, the cost per discounted death averted was KW435.2 million (US\$334,787), and per discounted LYG was KW150.2 million (US\$115,549). For the 3-dose schedule, the cost per discounted death averted was KW301 million (US\$231,538) and per discounted LYG was KW103.9 million (US\$79,995). 
Economic Evaluation of Childhood 7-Valent Pneumococcal Conjugate Vaccination in Korea

FIGURE 2 Univariate Sensitivity Analyses at the Vaccine Price KW70,000 per Dose

Annual incidence of pneumonia (per 100,000)

Annual incidence of otitis media (per 100,000)

Annual incidence of bacteremia (per 100,000)

Discount rate for outcomes and cost

Annual incidence of meningitis (per 100,000)

Medical cost for pneumonia

Medical cost for otitis media

Mediical cost for bacteremia

Medical cost for meningitis
14,985

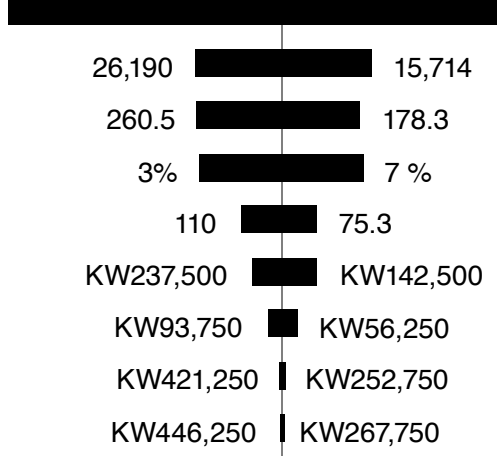

400

500

600

ICER (KW millions per LYG)

$I C E R=$ incremental cost-effectiveness ratio; $K W=$ Korean won; $L Y G=$ life years gained.

\section{Sensitivity Analyses}

Results of the univariate sensitivity analyses are shown in Figure 2. The ICER was decreased by reducing the vaccine cost and increasing disease incidence rates in the sensitivity analyses. The annual incidence of pneumonia appeared to be the disease parameter to which the ICERs were most sensitive. As expected, the ICERs were more favorable when the cost of vaccine was lower. At vaccine prices of KW30,000 and KW20,000 per dose, the corresponding ICERs per discounted death averted were KW71 million and KW20 million, respectively. The break-even costs at which the acquisition cost of vaccine equals the discounted cost offsets from vaccine were KW22,100 and KW28,100 for the 4- and 3-dose schedules, respectively (Figure 3). In probabilistic sensitivity analyses, mean ICERs were KW495 million (95\% CI=392-550) and KW324 million (95\% CI=222-373) in the 4- and 3-dose scenarios, respectively (data not shown).

The acceptability curves for a universal vaccination program at various vaccine price cut-offs and dosing schedules are shown in Figure 4. With a 4-dose schedule and 50\% probability of vaccination being cost-effective, values for WTP for an additional LYG were KW18 million, KW116 million, and KW310 million at vaccine prices per dose of KW20,000, KW30,000, and KW50,000, respectively. As expected, the probabilities of vaccination being cost-effective in the 3-dose schedule were higher than in the 4-dose schedule at the same WTP.

\section{Discussion}

Although decisions about universal introduction of a vaccine to a national child immunization program are strongly influenced by the vaccine's acquisition price, an overall evaluation of both the costs and benefits of a program is necessary for an informed decision. In this study, a decision analytic model estimated that a universal PCV-7 program would significantly reduce pneumococcal disease incidence and mortality for 5 years after birth. The study also demonstrated the potential to offset some medical and nonmedical costs with vaccination. Study results may be helpful for decision makers to determine whether or not to introduce PCV-7 to the universal vaccination program in Korea. As the KCDC continues to evaluate immunization programs, a costeffectiveness study of PCV-7 may provide a useful framework for assessing the health and economic impact in Korea.

Decision analytic models similar to that reported in the present study were used in economic evaluations for PCV-7 in the United States, ${ }^{19}$ Australia, ${ }^{22}$ Finland,${ }^{23}$ and the Netherlands, ${ }^{58}$ and for the Haemophilus influenzae type B (Hib) vaccine in Korea. ${ }^{59}$ This type of model can be used to compare the cost-effectiveness of a vaccination with no vaccination option as newer vaccines are introduced.

The economic benefits of PCV-7 vaccination have been evaluated in a number of countries. The results have been used to inform vaccine policy change decisions, sometimes to include PCV-7 in the country's national immunization program. These studies are diverse in their assumptions about vaccine efficacy, incidence rates, and reported results. In a 2000 study of the projected cost savings associated with PCV-7 in the United States, it was estimated that vaccination of infants would result in net savings for society and health care payers if the vaccine cost per dose was less than $\$ 46$ and $\$ 18$ respectively. ${ }^{19}$ In a 2004 cost-effectiveness study conducted in England and Wales, the universal vaccination program was not expected to be cost-effective from 


\section{FIGURE 3 ICER for PCV-7 Vaccination Program Depending on} Vaccine Price per Dose in Different Dosing Schedules

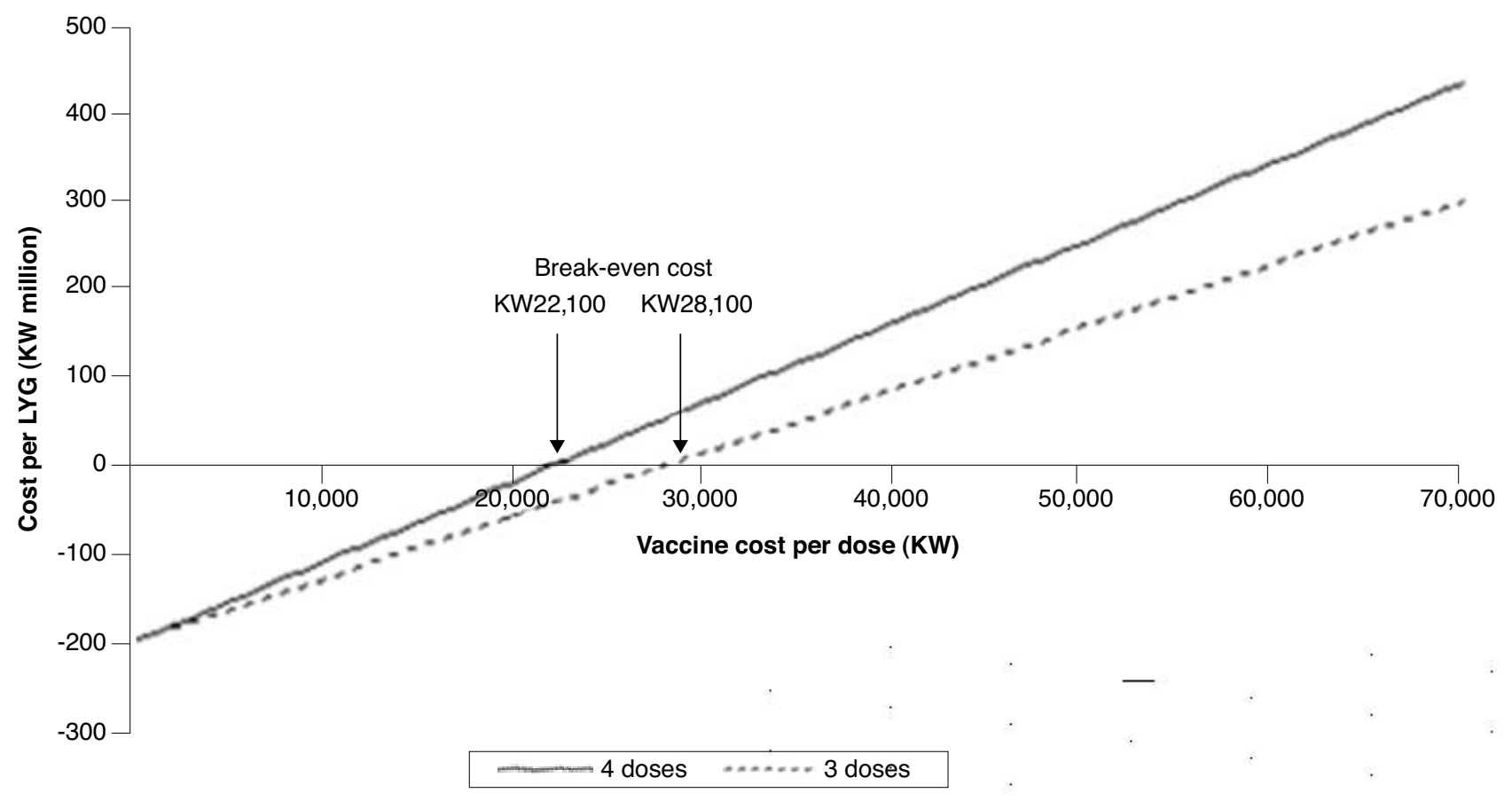

ICER=incremental cost-effectiveness ratio; $K W=$ Korean won; $L Y G=$ life years gained; $P C V-7=7$-valent pneumococcal conjugate vaccine.

the National Health Service perspective at the current price of the vaccine (assumed 30 pounds (£) per dose, 3 -dose program); however, researchers estimated that a 50\% reduction in vaccine cost would bring the cost per quality-adjusted life year (QALY) gained to within normal acceptable ranges ${ }^{60}$ In a 2003 Canadian study, it was estimated that the societal benefit-to-cost ratio would be 0.57 at the vaccine price of $\$ 58$ per dose, and vaccination would result in net savings for society if the vaccine price was less than $\$ 30$ per dose. $^{61}$ In the Netherlands, it was reported that the baseline cost-effectiveness ratio of a PCV-7 vaccination program at a vaccine price of 40 euros $(€)$ per dose would be relatively unfavorable when compared with other interventions that have been implemented in the country. However, after adjustment for the incidence rates of meningitis and bacteremia and inclusion of the herd protection effect (i.e., reduction in the disease incidence rate from reduced transmission of pneumococci after infant vaccination to nonvaccinated individuals), ${ }^{62}$ the ICER was reduced by one-fourth. ${ }^{58,63}$ In these studies, vaccine purchasing cost was found to be the most important variable that affects the decision whether to include PCV-7 in the national immunization program in individual countries. ${ }^{2}$

Several factors other than those assessed in the present study have the potential to affect the cost-effectiveness of a vaccina- tion program. A review of recently published economic evaluation studies for PCV-7 programs conducted during 2002-2006 showed that cost-effectiveness of $\mathrm{PCV}-7$ vaccination programs would be viewed as attractive in developed countries if the net long-term impact remains beneficial, determined by a mixture of effects related to herd immunity, serotype replacement (i.e., change in serotype distribution occurring after infant vaccination), ${ }^{60}$ and antibiotic resistance. Cost-effectiveness of PCV-7 vaccination would also be enhanced if a 3-dose schedule confers near-equivalent protection to a 4 -dose schedule. ${ }^{2}$ The costeffectiveness of PCV-7 has recently been updated to include the indirect effects of herd immunity on incidence of invasive pneumococcal disease, pneumonia requiring hospitalization, and otitis media after 7 years of use in the United States. The ICER dropped from US $\$ 201,000$ per life year saved to US $\$ 10,400$ per life year saved after accounting for herd immunity. ${ }^{62}$ In summary, the benefits of vaccine programs are difficult to evaluate precisely because of uncertainties about the current pneumococcal disease burden, period of vaccine efficacy, and the long-term effect of vaccines on pneumococcal disease epidemiology. Some assumptions of our model should be verified using clinical data in the future to reduce uncertainties.

PCV-7 vaccination may have other benefits, such as reducing 


\section{FIGURE 4 Acceptability Curve of PCV-7 Vaccination Program Depending} on Vaccine Price per Dose and Dosing Schedule

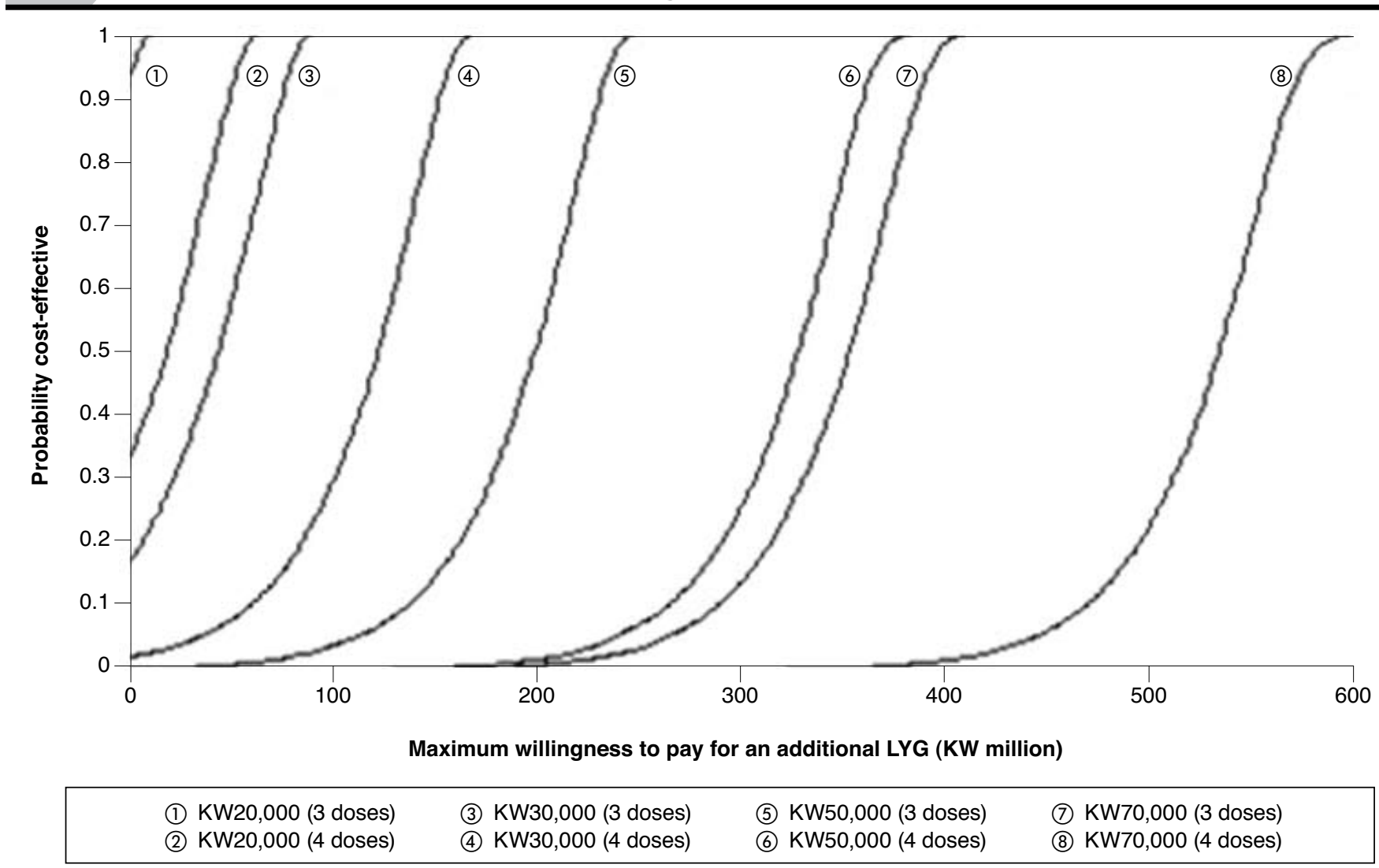

$K W=$ Korean won; $L Y G=$ life year gained; $P C V-7=7$-valent pneumococcal conjugate vaccine.

antibiotic-resistant $s p$ organisms. Korea is an area with serious antibiotic resistance problems. There is some evidence to suggest that $s p$ isolates resistant to antibiotics were reduced by PCV-7 vaccination. ${ }^{64}$ As antibacterial resistance is a risk factor for $s p$ causative infections, reduction of antibiotic-resistant pathogens could have additional positive effects not measured in the current analysis. On the other hand, PCV-7 has preventive effects for only about 90 sp specific serotypes, but these can be replaced by other serotypes not included in a vaccine after introduction of vaccination. Invasive pneumococcal diseases caused by serotypes other than those found in PCV-7 were found to be increased during the vaccine period (2002-2006) compared with the pre-vaccine period (1997-2001) in Spain. ${ }^{65}$ Serotype replacement has emerged as a limitation of PCV-7 but is still controversial. Furthermore, if serotype replacement caused a reduction in the carriage of vaccine serotypes, herd immunity would be of less interest. Also, there are some variations in common pneumococcal serotypes in different parts of the world and in transmission of the pathogen in different socio-cultural circumstances. These variations mean that overall and long-term effectiveness of widespread use of PCV-7 is difficult to predict at the population level. In addition, as there were no data in Korea to estimate the potential effects of herd immunity and a lack of reliable information on the changes of vaccine efficacy from decreased antibacterial resistance, we analyzed only direct vaccine effectiveness for the first 5 years in a birth cohort without modeling the possibility of serotype replacement or herd immunity.

The main health outcomes measured in this analysis were deaths averted and LYG on the basis of reduced mortality. We did not capture quality of life (QOL) gains attributable to the reduced short- and long-term morbidity associated with pneumococcal disease because we had no available QOL data in Korean children for the individual health states considered in this model. It is not possible to quantify the impact of this omission on the resulting ICERs and their apparent attractiveness. In a study by Butler et al. (2004), ${ }^{22}$ the ICER for PCV-7 was AUS\$230,130 per LYG, while the cost per disability-adjusted life year (DALY) averted was AUS $\$ 121,100$. In a study by Hubben et al. (2007), ${ }^{63}$ the ICER for 
PCV-7 was $€ 15,600$ per LYG and $€ 14,000$ per QALY gained. In both cases the ICER was more attractive when a QOL adjustment was made to the health outcome in the ratio.

An economic evaluation of a Hib vaccination program by Shin et al. (2008) conducted in Korea can be used as a reference to compare with the present study's results. Shin et al. found that the benefit-to-cost ratio for Hib vaccination was 0.77 at the assumed vaccination cost of KW26,000, but the ratio would be increased to 1.0 if the vaccination cost was less than KW20,000, one-half the current price of KW40,000 per dose of vaccine. ${ }^{59}$ Shin et al. suggested that the low economic benefit was attributable to the low incidence rate of Hib infection and the high price of the Hib vaccine.

Our study results show that a nationwide PCV-7 vaccination program would produce a net savings if the vaccine prices per dose were no more than KW22,100 (US\$17) for a 4-dose or 28,100 (US\$22) for a 3-dose regimen, suggesting that similar price levels for both Hib and PCV-7 are appropriate for integration into a universal immunization program. The public health rationale should be clearly based on clinical outcomes, benefits, and costs. A decision about PCV-7 introduction into immunization programs would depend on vaccine price as a major determinant of cost-effectiveness.

\section{Limitations}

First, we used a simplified decision analytic model to avoid too many assumptions. For the disease sequelae, some events may occur in combination, or more rare events may occur that were not captured in the model.

Second, vaccine efficacy was followed for only 5 years after birth even though the duration of efficacy may be longer. Longer follow-up would produce much better clinical outcomes and cost savings because input cost would not increase while outcomes, including reduced productivity loss and disease incidence for the extended period, would be counted. This limitation might make the results of our analysis conservative. Also, only confirmed cases proven from culture were assumed in this study, potentially resulting in underestimation of actual pneumococcal disease incidence.

Third, there are uncertainties and potential biases in the disease incidence rates and vaccine efficacy rates used in this model. In some instances, we used data from other countries because Korean epidemiology data were not available. We estimated otitis media incidence on a per patient basis from NHI data rather than a per episode basis, resulting in an underestimation of disease burden and efficacy. The study by Lee et al. (2006) ${ }^{1}$ that was used to estimate disease-related morbidities and mortalities was a 10-year retrospective study conducted in university hospitals. This setting is highly selective, and high-risk patients are usually referred to teaching hospitals, many of which are in a localized geographic area. In spite of these shortcomings, our use of Korea- specific data for most key parameters provided a reasonably accurate assessment of invasive pneumococcal diseases in Korean children. For vaccine efficacy, data from other countries were used when Korean data were not available. However, we adjusted assumptions about PCV-7 vaccine efficacy to reflect the distribution of serotypes most prevalent in Korea.

Fourth, because assumptions about pneumococcal diseaserelated direct medical costs were derived from $\mathrm{NHI}$ data, ${ }^{37}$ they did not include costs paid by patients, resulting in an underestimation of the cost of disease. The break-even costs for vaccine would have been higher if patient out-of-pocket expenditures were included in the cost offsets of a vaccination program. In order to better understand the impact that these uncertainties may have on outcomes, sensitivity analyses were performed for varied ranges of pneumococcal disease incidence rates and cost of disease. The ICERs were most sensitive to the incidence of pneumonia and the cost of vaccine.

Finally, we did not consider some factors that affect vaccine efficacy and the magnitude of its benefits on a population basis. These include the indirect effect or herd immunity protection inferred on unvaccinated individuals of all ages, the potential to reduce antibiotic resistance due to decreased use of antibiotics, and replacement of PCV-7 serotypes with nonvaccine serotypes. No reliable Korean data were available on these factors. PCV-7 has been reported to have the potential to reduce nasopharyngeal colonization and transmission of vaccine-type pneumococcal pathogens from vaccinated children to the unvaccinated population. ${ }^{5,66}$ This herd immunity effect could also have an impact on the cost-effectiveness of the PCV-7 strategies. There is some evidence that pneumococcal disease incidence is lower in both children and adults since universal PCV-7 vaccination was introduced in children. ${ }^{67-69}$

\section{Conclusion}

In this analysis, we predicted that universal PCV-7 vaccination of infants in Korea can significantly reduce the economic burden, morbidity, and mortality of pneumococcal disease by preventing pneumococcal infections. However, the cost-effectiveness of either a 4- or 3-dose vaccination strategy based on deaths averted or LYGs appears to be unattractive at current vaccine prices. The literature suggests that factors not considered in this analysis have the potential to make the public health impact and cost-effectiveness of universal PCV-7 vaccination in Korea more favorable. The impact of herd immunity, improved quality of life among nonfatal pneumococcal disease patients, reduced antibiotic resistance, longer than assumed duration of efficacy, and potential confounding of the true incidence rate because of empiric antibiotic therapy could improve the ICERs and net costs. Future research is needed to improve knowledge about these parameters in Korea and better inform future cost-effectiveness modeling. 


\section{Authors}

HYUN SOON SOHN, PhD, is Lecturer, College of Pharmacy, Sookmyung Women's University, Seoul, South Korea. EUNJIN JANG, $\mathrm{PhD}$, is Principal Researcher, Outcomes Research Team, National Evidence-Based Healthcare Collaborating Agency, Seoul, South Korea. DONG-CHURL SUH, MBA, PhD, is Professor; and JIN-WON KWON, PhD, is Research Fellow, Ernest Mario School of Pharmacy, Rutgers, The State University of New Jersey, Piscataway, New Jersey.

AUTHOR CORRESPONDENCE: Dong-Churl Suh, MBA, PhD, Department of Pharmacy Practice and Administration, Ernest Mario School of Pharmacy, Rutgers, The State University of New Jersey, 160 Frelinghuysen Rd., Piscataway, NJ 08854-8020. Tel: 732.445.5215, Ext. 402; Fax: 732.445.2533; Email: dsuh@rci.rutgers.edu.

\section{DISCLOSURES}

This research was performed in a university setting without external funding. Study concept and design were contributed primarily by Sohn and Kwon with the assistance of Suh. Data collection was performed primarily by Sohn with the assistance of Kwon. Data interpretation and writing of the manuscript were performed primarily by Sohn and Jang. Sohn and Suh made most of the manuscript revisions.

\section{REFERENCES}

1. Lee HJ, Kim KH, Chun BC, Choi EH. Disease burden of pneumococcal infection and serotypes of Streptococcus pneumoniae in Korean children. 2006. Korean Centers for Disease Control and Prevention.

2. Beutels P, Thiry N, Van Damme P. Convincing or confusing? Economic evaluations of childhood pneumococcal conjugate vaccination-a review (2002-2006). Vaccine. 2007;25(8):1355-67.

3. Kim SM, Hur JK, Lee KY, et al. Epidemiological study of pneumococcal nasal carriage and serotypes among Korean children. Korean J Pediatr. 2004:47(6):611-16

4. Sohn YM. Use of vaccine in the era of antimicrobial resistance: need of effective pneumococcal vaccines. Yonsei Med J. 1998;39(6):611-18.

5. Black S, Shinefield H, Fireman B, et al. Efficacy, safety and immunogenicity of heptavalent pneumococcal conjugate vaccine in children. Northern California Kaiser Permanente Vaccine Study Center Group. Pediatr Infect Dis J. 2000;19(3):187-95.

6. Eskola J, Kilpi T, Palmu A, et al. Efficacy of a pneumococcal conjugate vaccine against acute otitis media. N Eng J Med. 2001;344(6):403-09.

7. Black SB, Shinefield HR, Ling S, et al. Effectiveness of heptavalent pneumococcal conjugate vaccine in children younger than five years of age for prevention of pneumonia. Pediatr Infect Dis J. 2002;21(9):810-15.

8. Fireman B, Black SB, Shinefield HR, Lee J, Lewis E, Ray P. Impact of the pneumococcal conjugate vaccine on otitis media. Pediatr Infect Dis J. 2003;22(1):10-16.

9. O'Brien KL, Moulton LH, Reid R, et al. Efficacy and safety of seven-valent conjugate pneumococcal vaccine in American Indian children: group randomised trial. Lancet. 2003;362(9381):355-61.

10. Kilpi T, Ahman H, Jokinen J, et al. Protective efficacy of a second pneumococcal conjugate vaccine against pneumococcal acute otitis media in infants and children: randomized, controlled trial of a 7-valent pneumococcal polysaccharide-meningococcal outer membrane protein complex conjugate vaccine in 1666 children. Clin Infect Dis. 2003;37(9)1155-64.
11. Centers for Disease Control and Prevention (CDC). Direct and indirect effects of routine vaccination of children with 7-valent pneumococcal conjugate vaccine on incidence of invasive pneumococcal disease-United States, 1998-2003. MMWR Morb Mortal Wkly Rep. 2005;54(36):893-97. Available at: http://www.cdc.gov/mmwr/preview/mmwrhtml/mm5436al.htm. Accessed December 9, 2009.

12. Lee HJ, Kang JH, Kim KH. Establishment of pneumococcal vaccination guidelines. 2005. Korean Centers for Disease Control and Prevention.

13. WHO position paper. Pneumococcal conjugate vaccine for childhood immunization. Wkly Epidemiol Rec. 2007;82(12):93-104. Available at: http:// www.who.int/wer/2007/wer8212.pdf. Accessed December 9, 2009.

14. Kang E, Kim K, Lee S, Hong K. Surveillance for antimicrobial resistance of Streptococcus pneumoniae among children of attending day care centers in Seoul, Korea. Korean J Clin Pathol. 2000;20(2):171-77.

15. Kim KH, Lee JE, Whang IT, et al. Serogroup and antimicrobial resistance of Streptococcus pneumoniae isolated from oropharynx in children attending day care center. Korean J Pediatr Soc. 2002;45(3):346-53.

16. Cho KY, Lee JA, Cho SE, et al. A study of serotyping of Streptococcus pneumoniae by multibead assay. Korean J Pediatr. 2007;50(2):151-56.

17. Choi EH, Kim SH, Eun BW, et al. Streptococcus pneumoniae serotype 19A in children, South Korea. Emerg Infect Dis. 2008;14(2):275-81.

18. Pneumococcal conjugate vaccine: cost. Medscape Bus Med. Available at: http://www.medscape.com/viewarticle/410906_4. Accessed December 9, 2009

19. Lieu TA, Ray GT, Black SB, et al. Projected cost-effectiveness of pneumococcal conjugate vaccination of healthy infants and young children. JAMA. 2000;283(11):1460-68

20. Korea National Statistical Office. Korean Statistical Information Service. Available at: http://www.kosis.kr. Accessed December 9, 2009.

21. Ess SM, Schaad UB, Gervaix A, Pinosch S, Szucs TD. Cost-effectiveness of a pneumococcal conjugate immunization program for infants in Switzerland. Vaccine. 2003;21(23):3273-81.

22. Butler JR, McIntyre P, MacIntyre CR, Gilmour R, Howarth AL, Sander B. The cost-effectiveness of pneumococcal conjugate vaccination in Australia. Vaccine. 2004:22(9-10):1138-49.

23. Salo H, Sintonen H, Nuorti JP, et al. Economic evaluation of pneumococcal conjugate vaccination in Finland. Scand J Infect Dis. 2005;37(11-12):82132 .

24. Kim JS, Jang YT, Kim JD, et al. Incidence of Haemophilus influenzae type $\mathrm{b}$ and other invasive disease in South Korean children. Vaccine. 2004:22(29-30):3952-62

25. Robinson KA, Baughman W, Rothrock G, et al. Epidemiology of invasive Streptococcus pneumoniae infection in the United States, 1995-1998: opportunities for prevention in the conjugate vaccine era. JAMA. 2001;285(13):172935.

26. Baraff LJ, Lee SI, Schriger DL. Outcomes of bacterial meningitis in children: a meta-analysis. Pediatr Infect Dis J. 1993;12(5):389-94.

27. Koomen I, Grobbee DE, Roord JJ, Donders R, Jennekens-Schinkel A, van Furth AM. Hearing loss at school age in survivors of bacterial meningitis: assessment, incidence, and prediction. Pediatrics. 2003;112(5):1049-53.

28. Goller Y. Cochlear implantation in children: implications for the primary care provider. J Am Acad Nurse Pract. 2006;18(9):397-408.

29. Kim CS, Kim DK, Suh MW, Oh SH, Chang SO. Clinical outcomes of cochlear reimplantation due to device failure. Clin Exp Otorhinolaryngol. 2008;1(1):10-14

30. Mattila PS. Adenoidectomy and tympanostomy tubes in the management of otitis media. Curr Allergy Asthma Rep. 2006;6(4):321-26.

31. Palmu AA, Verho J, Jokinen J, Karma P, Kilpi TM. The seven-valent pneumococcal conjugate vaccine reduces tympanostomy tube replacement in children. Pediatr Infect Dis J. 2004;23(8):732-38. 
32. Lee YJ, Lee SJ, Park HJ, et al. A clinical study of child bacterial meningitis in Daejeon and Chungcheong area: 2001-2005. Korean J Pediatr. 2007;50(2):156-62

33. Ahn JH, Lee JY, Kim Y, Kim YI, Shim HJ, Lee KS. Analysis of necessity and adequateness of hearing rehabilitations in hearing impaired persons. Korean J Otolaryngol. 2007;50(3):198-202.

34. Park DC, Choi IS, Heo JH, Lee KW. Acute bacterial meningitis: causative organisms, clinical characteristics and prognosis. J Korean Neurol Assoc. 2000;18(5):556-61.

35. Kim HT, Jang HO, Moon JS, et al. Etiology of community-acquired bacteremia in healthy children. Korean J Pediatr. 2005;48(7):716-22.

36. Choi EH, Lee HJ. Clinical outcome of invasive infections by penicillinresistant Streptococcus pneumoniae in Korean children. Clin Infect Dis. 1998;26(6):1346-54.

37. Health Insurance Review \& Assessment Service Agency. National Health Insurance Statistical Yearbook. Seoul, South Korea: HIRA; 2006.

38. Lee SO, Woo JH. Epidemiology and etiology of community-acquired pneumonia. J Korean Med Assoc. 2007;50(10):860-67.

39. Jung DS, Kim HS, Park CW, Oh SH. The causative organisms of otitis media accompanying otorrhea in children and their antimicrobial susceptibility. Korean J Pediatr Infect Dis. 2000;7(2):233-39.

40. Lee YJ, Lee WS, Kim KS, Kim KB. Prognosis of bacterial meningitis based on cerebrospinal fluid culture. J Korean Pediatr Soc. 1998;41(5):614-21.

41. Choi EH. Direct and indirect effects of pneumococcal protein conjugate vaccine. J Korean Med Assoc. 2008;51(2):119-26.

42. Lee JA, Kim NH, Park KW, et al. Serotypes and penicillin susceptibility of Streptococcus pneumoniae isolated from clinical specimens and healthy carriers of Korean children. J Korean Pediatr Soc. 2003;46(9):846-53

43. Choi KM, Yeon SI, Shin JS, Yong DE, Lee KW, Kim DS. Serotype and antimicrobial susceptibility of Streptococcus pneumoniae. Infect Chemother. 2006;38(4):179-85.

44. Korea Center for Disease Control and Prevention. Vaccine information. Available at: http://cdc.go.kr/kcdchome/jsp/home/health/vac/ HEALVAC0001Detail.jsp? menuid=100182\&contentid=4020\&boardid=null $\&$ appid $=$ kcdchome $\&$ pageNum $=3 \&$ sub $=4 \&$ tabin $x=2 \& q \_$had01 $=$A\&q_had02 $=$

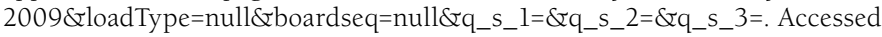
December 9, 2009

45. Kayhty H, Ahman H, Eriksson K, Sorberg M, Nilsson L. Immunogenicity and tolerability of a heptavalent pneumococcal conjugate vaccine administered at 3, 5 and 12 months of age. Pediatr Infect Dis J. 2005;24(2)108-14.

46. Esposito S, Pugni L, Bosis S, et al. Immunogenicity, safety and tolerability of heptavalent pneumococcal conjugate vaccine administered at 3,5 and 11 months post-natally to pre- and full-term infants. Vaccine. 2005;23(14):1703-08.

47. Belgian Health Care Knowledge Centre. Effects and costs of pneumococcal conjugate vaccination of Belgian childrens. KCE Reports Vol. 33C. 2006 Available at: http://kce.fgov.be/index_en. aspx?SGREF=5223\&CREF=8069. Accessed December 9, 2009.

48. Kim NH, Lee J, Lee SJ, et al. Immunogenicity and safety of pneumococcal 7-valent conjugate vaccine (diphtheria CRM(197) protein conjugate; Prevenar) in Korean infants: differences that are found in Asian children. Vaccine. 2007:25(45);7858-65.

49. Whitney CG, Pilishvili T, Farley MM, et al. Effectiveness of seven-valent pneumococcal conjugate vaccine against invasive pneumococcal disease: a matched case-control study. Lancet. 2006;368(9546):1495-502.

50. De Graeve D, Beutels P. Economic aspects of pneumococcal pneumonia: a review of the literature. Pharmacoeconomics. 2004;22(11):719-40.

51. Eskola J, Anttila M. Pneumococcal conjugate vaccines. Pediatr Infect Dis J. 1999;18(6):543-51.
52. ezday. Immunization costs. Available at: http://www.ezday.co.kr/bbs/ view_board.html?q_id_info=370\&q_sq_board=527400. Accessed December 9, 2009.

53. Ministry of Health and Welfare. National Health Insurance Reimbursement Price List. Seoul, South Korea: Korean Medical Association; 2006.

54. Ministry of Health and Welfare. The third Korea National Health and Nutrition Examination Survey. Seoul, South Korea: Ministry of Health and Welfare; 2005.

55. Park SE, Lee H, Lim SY, Kim KY. Immunogenicity of 7-valent pneumococcal conjugate vaccine related to booster immunization in Korean children. Korean J Pediatr. 2008;51(6):622-28.

56. Korea Pharmaceutical Traders Association. Yearbook of Imported Pharmaceutical Goods. Seoul, South Korea: Korea Pharmaceutical Traders Association; 2006.

57. Briggs A, Sculpher M, Claxton K. Further development in decision analytic models for economic evaluation. In: Briggs A, Claxton K Sculpher M, (eds). Decision Modelling for Health Economic Evaluation. New York: Oxford University Press; 2006:45-76.

58. Bos JM, Rümke H, Welte R, Postma MJ. Epidemiologic impact and cost-effectiveness of universal infant vaccination with a 7-valent conjugated pneumococcal vaccine in the Netherlands. Clin Ther. 2003;25(10):2614-30.

59. Shin S, Shin YJ, Ki M. Cost-benefit analysis of Haemophilus influenza type B immunization in Korea. J Korean Med Sci. 2008;23(2):176-84.

60. Melegaro A, Edmunds WJ. Cost-effectiveness analysis of pneumococcal conjugate vaccination in England and Wales. Vaccine. 2004;22(31-32):420314.

61. De Wals P, Petit G, Erickson LJ, et al. Benefits and costs of immunization of children with pneumococcal conjugate vaccine in Canada. Vaccine. 2003;21(25-26):3757-64

62. Ray GT, Pelton SI, Klugman KP, et al. Cost-effectiveness of pneumococcal conjugate vaccine: an update after 7 years of use in the United States. Vaccine. 2009;27(47):6483-94.

63. Hubben GA, Bos JM, Glynn DM, et al. Enhanced decision support for policy makers using a web interface to health-economic models-illustrated with a cost-effectiveness analysis of nation-wide infant vaccination with the 7-valent pneumococcal conjugate vaccine in the Netherlands. Vaccine. 2007;25(18):3669-78

64. Dagan R, Sikuler-Cohen M, Zamir O, et al. Effect of a conjugate pneumococcal vaccine on the occurrence of respiratory infections and antibiotics use in day-care center attendees. Pediatr Infect Dis J. 2001(10);20:951-58.

65. Muñoz-Almagro C, Jordan I, Gene A, Latorre C, Garcia-Garcia JJ, Pallares R. Emergence of invasive pneumococcal disease caused by nonvaccine serotypes in the era of 7 -valent conjugate vaccine. Clin Infect Dis. 2008;46(2):174-82

66. Poehling KA, Talbot TR, Griffin MR, et al. Invasive pneumococcal disease among infants before and after introduction of pneumococcal conjugate vaccine. JAMA. 2006;295(14):1668-74

67. Whitney CG, Farley MM, Hadler J, et al. Decline in invasive pneumococcal disease after the introduction of protein-polysaccharide conjugate vaccine. N Engl J Med. 2003;348(18):1737-46. Available at: http://content.nejm. org/cgi/reprint/348/18/1737.pdf. Accessed December 9, 2009.

68. Lexau CA, Lynfield R, Danila R, et al. Changing epidemiology of invasive pneumococcal disease among older adults in the era of pediatric pneumococcal conjugate vaccine. JAMA. 2005;294(16):2043-51. Available at: http://jama.ama-assn.org/cgi/reprint/294/16/2043. Accessed December 9, 2009 .

69. Centers for Disease Control and Prevention (CDC). Invasive pneumococcal disease in children 5 years after conjugate vaccine introduction-eight states, 1998-2005. MMWR Morb Mortality Wkly Rep. 2008;57(6):144-48. Available at: http://www.cdc.gov/mmwr/preview/mmwrhtml/mm5706a2. htm. Accessed December 9, 2009. 